\title{
Comparison of intestinal permeability of nebivolol hydrochloride loaded solid lipid nanoparticles with commercial nebivolol tablet
}

\author{
Evren Homan GÖKÇE 1 *, Mustafa Sinan KAYNAK ${ }^{2}$, Aysu YURDASIIPER 1 , \\ Neslihan ÜSTÜNDAĞ-OKUR ${ }^{3}$, Selma ŞAHİN ${ }^{4}$
}

1 Department of Pharmaceutical Technology, Faculty of Pharmacy, Ege University, 35100, İzmir, Turkey.

2 Department of Pharmaceutical Technology, Faculty of Pharmacy, İnönü University, Malatya, Turkey.

3 Department of Pharmaceutical Technology, School of Pharmacy, İstanbul Medipol University, 34810, İstanbul, Turkey.

4 Department of Pharmaceutical Technology, Faculty of Pharmacy, Hacettepe University, 06100, Ankara, Turkey.

* Corresponding Author. E-mail: evrenhomangokce@gmail.com (E.H.G.); Tel. +90-232-311 40 67; ORCID No: 0000-00020751-5563.

Received: 15 February 2018 / Revised: 16 April 2018 / Accepted: 26 April 2018

ABSTRACT: The oral application of drugs is the most popular route for achieving systemic effects, nevertheless, it is limited by difficulties related to physicochemical properties of the drug. Solid lipid nanoparticles (SLNs) are appealing extensive notice because of showing increased solubility and improved oral bioavailability via different mechanisms. The aim of the study is to compare and peruse the in-situ permeation of nebivolol (NBV) loaded SLN and its commercial tablet formulation used for the treatment of hypertension. For this aim Single-Pass Intestinal Perfusion (SPIP) method was used for in-situ permeation studies. NBV loaded SLNs were prepared and modified with polyethylene glycol (PEG). In order to prepare SLNs by homogenization technique, compritol, lecithin and poloxamer were chosen. Particle sizes of blank and loaded SLN were $213.4 \pm 17.5$ and $264.1 \pm 18.8 \mathrm{~nm}$, respectively with polydispersity index values of approximately 0.3 for each. NBV loading resulted in positive electrical charge on SLNs. The encapsulation efficiency was $98.04 \pm 0.2 \%$. Permeability coefficient values were tripled when NBV was incorporated in SLNs and doubled when pure NBV was given separately with a blank SLN. PEG modified SLN can be used to enhance oral absorption of NBV, and SLNs alone can be used as permeation enhancer in oral drug delivery.

KEYWORDS: Solid lipid nanoparticle; nebivolol; segmental permeability; intestinal absorption.

\section{INTRODUCTION}

Hypertension, which is high blood pressure, can be counted as a main risk factor for cardiovascular diseases. Studies showed that there is a direct relationship between cardiovascular diseases such as stroke and coronary artery disease and the level of the high blood pressure [1,2]. The use of beta adrenergic receptor blockers is taking attention as first-line treatment of all types of arterial hypertension [3,4].

Nebivolol (NBV) is a third generation cardioselective beta 1-blocker which provides combination of beta-adrenergic blocking activity and vasodilation. Furthermore, NBV can be used for the treatment of angina cordis and congestive heart failure as an addition to its usage as an antihypertensive agent [5]. This active substance, indeed, is currently being chosen for treatment of arterial hypertension because the way it reduces the blood pressure via vasodilatory properties mediated by the l-arginine/NO pathway [4, 6]. Currently, $10 \mathrm{mg}$ oral tablet form of NBV is available on the market.

Oral route is the most favored route for drug administration due to ease of application with high patient compliance. Nevertheless, in oral drug delivery there is a need for innovative drug delivery systems due to low drug solubility, poor gastrointestinal absorption, metabolism related variability issues, continuous fluctuation of drug plasma levels and food effects [7, 8]. Nebivolol is absorbed rapidly after oral administration and is not affected by food; however, it has variable absolute oral bioavailability due to its extensive first-pass metabolism [9]. Due to the low aqueous solubility and high membrane permeability, NBV is categorized as a Class II drug according to Biopharmaceutics Classification System [10]. Therefore, in

How to cite this article: Gokce EH, Kaynak MS, Yurdasiper A, Ustundag-Okur N, Sahin S. Comparison of intestinal permeability of nebivolol hydrochloride loaded solid lipid nanoparticles with commercial nebivolol tablet. Marmara Pharm J. $2018 ; 22$ (4): $578-586$. 
order to overcome the problems seen by conventional NBV tablets, an efficient oral drug delivery system is needed.

In order to increase the oral bioavailability, colloidal systems, especially nanoparticles are of particular importance. In case of nanoparticles, bioavailability depends on the properties of the nanocarrier instead of physicochemical properties of the drug [11-13]. Recently, solid lipid nanoparticles (SLNs) have increased attention due to their inimitable structure and properties, such as improved biocompatibility, high bioavailability, protection for the incorporated drug against degradation, and controlled release of drugs [14, 15]. As carriers, the artificial or natural synthetic solid lipids were used to obtain solid colloidal particles [16, 17]. SLNs are able to increase the bioavailability of lipophilic and poorly hydrophilic drugs. An antihypertensive drug nitrendipine's low oral bioavailability (10 to 20\%) was increased up to three- to four fold compare to that of a nitrendipine suspension, when formulated as SLN [18]. Beside these advantages, surface modification of SLNs with polyethylene glycol (PEG) lead to a developed surface hydrophilicity of nanoparticles which emerged improved transmucosal transport for drug delivery [19].

In oral delivery, the main absorption takes place in the small intestines, and absorption of drugs designed for oral applications could be different in duodenum, jejunum, and ileum. [20, 21]. In the formulation development, knowing the absorption site and absorption characteristics of active ingredient is important [22]. Since intestinal transit time is about 3-4 hours, it can be accepted as that the absorption of the drug is high in the entire intestine. In the light of this knowledge, for identification of development strategies of nanoparticulate formulations, absorption properties in the all intestinal tract with low solubility and/or low permeability drugs have a significant importance [23].

The main objective of the study was to compare and evaluate the in-situ permeation of NBV loaded SLN and its commercial tablet used for the treatment of hypertension. NBV loaded and PEG modified SLNs were developed to increase NBV's oral bioavailability. In order to prepare SLNs by homogenization technique, compritol, lecithin and poloxamer were selected. For this aim, Single-Pass Intestinal Perfusion (SPIP) method was used for in-situ permeation studies [21-25].

\section{RESULTS AND DISCUSSION}

In this study, SLNs were produced and evaluations were performed to determine the permeability characteristics of NBV as pure powder, in tablet and SLN formulations across the gastrointestinal tract.

\subsection{Preparation and characterization of SLNs}

NBV loaded SLNs were prepared by using high shear mixing followed by high pressure homogenization at 500 bars for 1 cycle. The characterization studies of the blank and NBV loaded SLNs were performed by measuring the particle size, polydispersity index (PDI), encapsulation efficiency and zeta potential.

Particle size distribution at nano level is very important for bioavailability enhancement in oral drug delivery. Particle size of unloaded SLN was $213.4 \pm 17.5 \mathrm{~nm}$. Loading NBV into SLNs made a significant increase in particle size $(264.1 \pm 18.8 \mathrm{~nm})(p<0.05)$.

Another important parameter is the measurement of PDI because these carriers are polydisperse in nature. PDI values between $0-0.3$ are monodisperse and homogeneous, but more than 0.3 is an indicator of non-homogeneity and Polydispersity [26]. PDI values obtained for blank and NBV loaded SLNs were $0.318 \pm 0.05$ and $0.309 \pm 0.05$, respectively.

A specific result was observed for zeta potential, since NBV gave a positive charge effect on SLNs. Addition of NBV changed zeta potential from approximately $-32 \mathrm{mV}$ to almost neutral $(-0.3 \mathrm{mV})$.

To calculate the entrapment efficiency of NBV, ultracentrifugation method was used. The encapsulation efficiency was found as $98.04 \pm 0.2 \%$, indicating the suitability of the formulation for NBV incorporation.

These results showed that NBV can be successfully encapsulated in SLNs with a high yield as a homogeneous distribution with the method employed in our study.

A detailed paper has been published about preparation, characterization, in vitro release and pharmacokinetic studies about NBV-SLNs by our team [17]. This study mainly focuses on in-situ permeation studies via SPIP method. 


\subsection{Permeability studies}

Metoprolol tartrate was used as a marker for the integrity of the experiment, and phenol red as a nonabsorbable marker in all groups. In order to determine NBV permeability, it is co-perfused with meteprolol tartrate, simultaneously. Results showed that permeability $\left(\mathrm{P}_{\text {eff }}\right)$ was $0.895 \pm 0.3 \mathrm{~cm} / \mathrm{sec}$ for metoprolol tartrate and $0.772 \pm 0.2 \mathrm{~cm} / \mathrm{sec}$ for NBV. $\mathrm{P}_{\text {eff }}$ values calculated for NBV following perfusion of ileum are shown in Figure 1.

The highest $P_{\text {eff }}$ and $k_{a}$ value was obtained with NBV-SLN and the least permeable forms were tablet and pure drug (Table 1). Net water flux (NWF) values indicated the water secretion into segment by pure NBV $(24.07 \mathrm{~mL} / \mathrm{h} / \mathrm{cm})$ and pure NBV administered together with blank SLN. A loss of fluid was observed from segment for NBV loaded SLN $(-21.43 \mathrm{~mL} / \mathrm{h} / \mathrm{cm})$ and NBV tablet $(-22.7 \mathrm{~mL} / \mathrm{h} / \mathrm{cm})$ administered groups. Yang et al. reported that the absorptive clearance values estimated for compounds from in situ perfusion studies is based on loss from the intestinal lumen, and require correction of effluent concentration with NWF. For a low permeability drug, an absorptive clearance estimated from a non-linear equation approaches to a linear relationship with NWF regardless of whether NWF has a real effect on absorptive clearance [27].

The increment in $\mathrm{P}_{\text {eff }}$ and $\mathrm{k}_{\mathrm{a}}$ was expected with NBV incorporation in SLNs. One study showed that loading andrographolide (antilipidemic drug) into SLNs enhanced the bioavailability of the drug as $241 \%$, compared to the drug's suspension [28]. Similarly, the SLN formulation increased the relative bioavailability of oxyresveratrol by $125 \%$ when compared with unformulated drug [29]. Our results were in accordance with these findings. $P_{\text {eff }}$ of NBV was increased by about $294 \%$ in our study, as expected. Moreover, it was surprising to find out that $\mathrm{P}_{\text {eff }}$ of NBV was doubled, when pure NBV and blank SLN were given together (Table 1). This result points out that SLN does not only serve as a colloidal matrix for encapsulated drugs but also acts like a permeation enhancer in oral drug delivery.

There are several possible mechanisms to explain the enhanced permeability/bioavailability of drugs given in nano-lipid carriers; such as i) maintaining a solubilized state of the drugs in the GI tract, ii) formation of mixed micelles, iii) promoting the secretion of endogenous phospholipids and bile salts [30]. These mechanisms might be used to explain the enhanced absorption obtained with NBV loaded SLNs. On the other hand, the increment in $\mathrm{P}_{\text {eff }}$ of the pure drug with SLN might be attributed to the muco-penetrating ability of SLN, and the enhanced intestinal lymphatic lipid flux. During this lipid flux pure drug absorption might be stimulated

Table 1. Absorption rate constant $\left(\mathrm{k}_{\mathrm{a}}\right)$ of metoprolol tartrate and NBVacross rat ileum.

\begin{tabular}{cc}
\hline Formulation & $\begin{array}{c}\text { Rat intestinal absorption } \\
\text { rate constant, } \\
\mathbf{k}_{\mathbf{a}}(\mathbf{x} \mathbf{1 0} \mathbf{- 4}, \mathbf{1} / \mathbf{s e c})\end{array}$ \\
\hline MTP & $9.72 \pm 3.5^{*}$ \\
NBV & $6.65 \pm 3.0^{*}$ \\
NBV + unloaded SLN & $8.98 \pm 3.1$ \\
NBV loaded SLN & $13.00 \pm 3.2$ \\
NBV (in tablet) & $6.39 \pm 3.0^{*}$ \\
\hline
\end{tabular}

* $\mathrm{p}<0.05$ as compared to "NBV loaded SLN" 


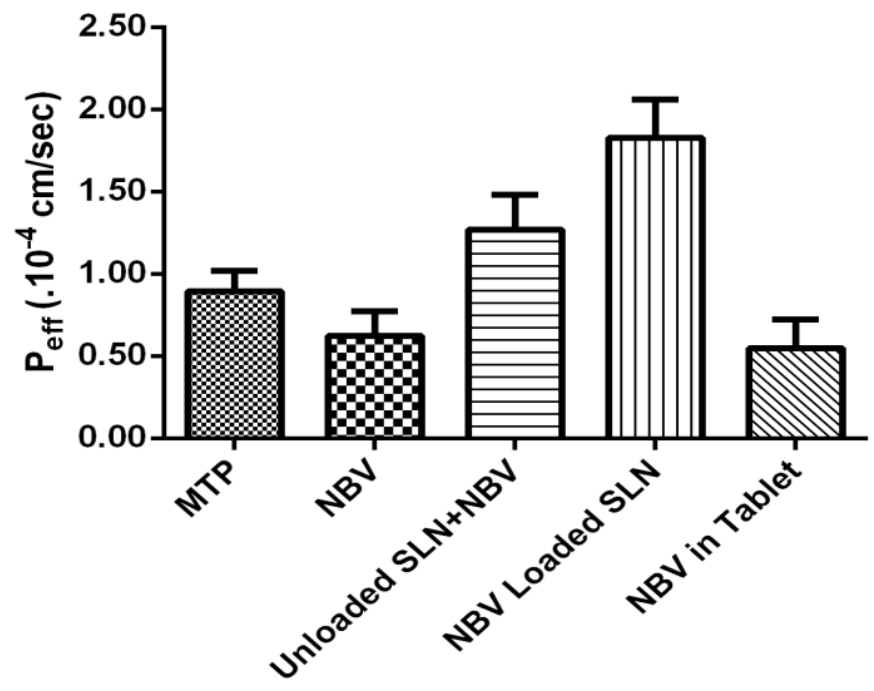

Figure 1. The permeability coefficients $\left(\mathrm{P}_{\text {eff, }} \mathrm{cm} / \mathrm{sec}\right)$ of NBV obtained from perfusion of rat ileum (mean $\pm S D ; n=6)$.

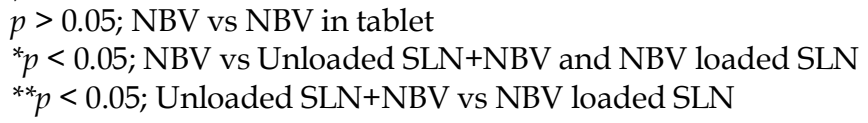

\section{CONCLUSION}

In this study, NBV loaded SLNs were successfully applied for determination of their permeation behaviour via in situ SPIP studies. Results show that PEG modified SLN can be used to enhance oral absorption of NBV, and SLNs alone can be used as permeation enhancer in oral drug delivery. However, there is still a need for the clarification of the mechanism for enhanced oral bioavailability, especially for lipid based colloidal systems.

\section{MATERIALS AND METHODS}

\subsection{Materials}

Metoprolol tartrate was donated from Novartis (Novartis Drug Co., Turkey). Ingredients of golytely solution $\left(\mathrm{NaCl}, \mathrm{KCl}, \mathrm{Na}_{2} \mathrm{SO}_{4}, \mathrm{NaHCO}_{3}\right.$ and mannitol) and ortho-phosphoric acid were supplied from SigmaAldrich (Germany). Phenol red was from Merck (Germany). Purified water $(18 \mathrm{M} \Omega / \mathrm{cm})$ was obtained by using New Human Power I water purification system (Korea). Poloxamer ${ }^{\circledR} 188$ (Pluronic F68) was donated by BASF (Germany). Compritol ${ }^{\circledR} 888$ was a kind gift from Gattefossée (France). NBV was from Sigma (Germany). PEG stearate (Simulsol M52) was obtained from Seppic (France). Soybean lecithin was supplied from Applichem (Germany). High pressure liquid chromatography (HPLC) grade acetonitrile was obtained from Sigma (Germany). All other chemicals and solvents were of HPLC or analytical reagent grade.

\subsection{Preparation of SLNs}

SLNs were obtained with high shear homogenization followed by high pressure technique optimized in our previous study [17]. Compritol 888 was used as lipid, lecithin and poloxamer 188 were employed as surfactant. The lipid phase (100 mg Compritol, $10 \mathrm{mg}$ lecithin and $100 \mathrm{mg}$ PEG) was melted at $70-80^{\circ} \mathrm{C}$. The aqueous phase $(100 \mathrm{mg}$ poloxamer) was dissolved in $15 \mathrm{~mL}$ ultrapure water and heated to the same temperature as the lipid phase. Under homogenization at $24.000 \mathrm{rpm}$ for $5 \mathrm{~min}$ with Ultra Turrax (T25, Ika Germany), the aqueous phase was transferred into the lipid phase. This mixture was dispersed in $10 \mathrm{~mL}$ ultrapure water at $4^{\circ} \mathrm{C}$. To improve particle size distribution, high pressure homogenizer (Microfluidizer / ML110L) was used at 500 bars for 1 cycle after SLNs were prepared with high shear homogenizer. For preparation of drug loaded SLNs and drug loaded modified SLNs, $1.5 \mathrm{mg}$ NBV was mixed with the lipid phase, melted together and prepared with the method as described above. 


\subsection{Characterization of SLNs}

The average particle size and PDI of NBV loaded SLNs were measured using dynamic light scattering method (Malvern Zetasizer Nano ZS, Malvern Instruments, U.K.) at an angle of $173^{\circ}$ at $25^{\circ} \mathrm{C}$. Before starting to the experiment, the samples were diluted with ultrapure water to have an appropriate scattering intensity. The viscosity of water was chosen as $0.0089 \mathrm{cp}$. The measurements were performed five times at $25^{\circ} \mathrm{C}$.

Plain folded capillary zeta cells (Malvern Zetasizer Nano ZS) was used for measurement of zeta potential. Electrophoretic mobility using Helmholtz-Smoluchowski Equation under an electrical field of 40 $\mathrm{V} / \mathrm{cm}$ was used to calculate the zeta potential of the samples. All measurements were repeated five times at $25^{\circ} \mathrm{C}$.

To evaluate the entrapment efficiency (EE \%), SLN and the free drug were separated by using ultracentrifugation. The non-encapsulated (free) NBV was determined by HPLC method. Following equation was used for estimation of $\mathrm{EE} \%$.

$\mathrm{EE} \%=\frac{\text { Total amount of NBV }- \text { Free NBV }}{\text { Total amount of NBV }} \times 100 \quad($ Eq. 1)

One $\mathrm{mL}$ of NBV loaded SLN formulation $(0.06 \mathrm{mg} / \mathrm{mL})$ was added in a dialysis bag (Spectro/por Dialysis Membrane, molecular weight cut-off of 12-14 kDa), placed in a centrifuge tube containing $9 \mathrm{~mL}$ of ultrapure water, and then centrifuged at $55.000 \mathrm{rpm}$ for $1.5 \mathrm{~h}$ (Beckman Coulter L100 XP-USA). Experiments were performed three times at $25 \pm 2^{\circ} \mathrm{C}$. The water phase was filtered through a membrane filter $(0.2 \mu \mathrm{m}$ Nylon, Milipore Millex-GN) and the amount of NBV in ultrapure water was determined by a HPLC system equipped with a UV detector (Dionex Ultimate 3000, USA).

NBV was determined by a Thermo Scientific HPLC system with UV dedector and Thermo Scientific C18 column (25-cm long, $4.5 \mu \mathrm{m}$ inner diameter) under the validated conditions: $\mathrm{pH} 3.0$ acetonitrile:phosphate dihydrogen buffer (50:50, v/v) used as the mobile phase. Flow rate was adjusted to $1.0 \mathrm{~mL} / \mathrm{min}$, and wavelength was $220 \mathrm{~nm}$. Volume of injection was $20 \mu \mathrm{l}$ and the column temperature was kept at $40^{\circ} \mathrm{C}$.

\subsection{In situ studies}

\subsubsection{Preparation of perfusion medium}

The perfusion medium was prepared using $40 \mathrm{mM} \mathrm{Na}_{2} \mathrm{SO}_{4}, 10 \mathrm{mM} \mathrm{KCl}, 25 \mathrm{mM} \mathrm{NaCl}, 80 \mathrm{mM}$ mannitol and $20 \mathrm{mM} \mathrm{NaHCO}_{3}$. Ortho-phosphoric acid was used to adjust the $\mathrm{pH}$ of buffer to 7.4. Before each experiment, freshly prepared perfusion medium was filtered via $0.45 \mu \mathrm{m}$ membrane filter.

\subsubsection{Single-pass intestinal perfusion (SPIP) studies}

Ethical permissions were obtained with 2014/A-61 protocol number from local ethic comitte (Committee of Use and Care of Animals, Inonu University), for animal studies. For intestinal perfusion studies, 200-300 g Sprague Dawley (female) rats were used. Before each experiment, animals were fasted for a period of 12-18 h but allowed to access to tap water. Animals were allocated one of the four groups given in Table 2. 
Table 2. Details of experimental groups.

\begin{tabular}{lll}
\hline Groups & \multicolumn{2}{l}{ Content of Reservoir Solutions } \\
\hline & Nebivolol (Pure) & $10 \mu \mathrm{g} / \mathrm{mL}$ \\
Group 1 & Metoprolol Tartrate & $400 \mu \mathrm{g} / \mathrm{mL}$ \\
& Phenol Red & $250 \mu \mathrm{g} / \mathrm{mL}$ \\
& Golytely Solution q.s & $100 \mathrm{~mL}$ \\
& & \\
& Nebivolol (Pure) & $10 \mu \mathrm{g} / \mathrm{mL}$ \\
& Blank SLN & $17 \mathrm{~mL}$ \\
Group 2 & Phenol Red & $250 \mu \mathrm{g} / \mathrm{mL}$ \\
& Golytely Solution q.s & $100 \mathrm{~mL}$ \\
& & \\
& NBV-SLN & $17 \mathrm{~mL}$ \\
& Phenol Red & $250 \mu \mathrm{g} / \mathrm{mL}$ \\
Group 3 & Golytely Solution q.s & $100 \mathrm{~mL}$ \\
& & \\
& NBV powdered tablet & $10 \mathrm{mg}$ \\
& Metoprolol Tartrate & $400 \mu \mathrm{g} / \mathrm{mL}$ \\
& Phenol Red & $250 \mu \mathrm{g} / \mathrm{mL}$ \\
& Golytely Solution q.s & $250 \mathrm{~mL}$ \\
&
\end{tabular}

In situ single pass intestinal perfusion test procedure used in this study was a modification of the previously published reports [21-25]. Under anaesthetizia $(60 \mathrm{mg} / \mathrm{kg}$ pentobarbital, intraperitoneal), the rats in a supine position were placed on an operation table at $37^{\circ} \mathrm{C}$. The abdomen was opened by a $3-4 \mathrm{~cm}$ midline incision. After cannulation of ileal segment $(7-10 \mathrm{~cm})$ with flexible PVC tubings (outlet tubing id 1.70 $\mathrm{mm}$ and inlet tubing id $0.76 \mathrm{~mm}$ ), it was attached to the perfusion system. Throughout the experiment, ileal segment moistened with saline, and covered with parafilm. The isolated ileal segment was rinsed for 10 minutes with blank perfusion solution $(0.5 \mathrm{~mL} / \mathrm{min})$ using a peristaltic pump (Minipuls 3, USA). The exposed ileal segment was perfused with Golytely solution containing the substance, phenol red and metoprolol tartrate (reference compounds) at a flow rate of $0.2 \mathrm{~mL} / \mathrm{min}$ for total period of $60 \mathrm{~min}$.

In perfusion studies, metoprolol tartrate was used as a high permeability reference compound in order to compare the permeability coefficient of pure NBV and NBV in tablet. Phenol red was used as a zero permeability marker in all groups for correction of permeability coefficients of tested drugs for water transport [24]. Metoprolol tartrate was not used in group 2 and group 3 because NBV $P_{\text {eff }}$ value in these groups (group 2 and group 3) was compared with the NBV $P_{\text {eff }}$ values in groups 1 and 4.

The oral dose was taken as reference to determine the concentrations $(0.01 \mathrm{mg} / \mathrm{mL}$ for NBV, 0.4 $\mathrm{mg} / \mathrm{mL}$ for metoprolol tartrate and $0.25 \mathrm{mg} / \mathrm{mL}$ for phenol red). Outflow samples were obtained in every 10 minutes intervals to eppendof tubes. The animals were sacrificed by cervical dislocation at the end of the experiments. Length of the perfused segment was noted.

\subsubsection{Data analysis}

$\mathrm{P}_{\text {eff }}$ was calculated after correcting measured $\mathrm{C}_{\text {out }} / \mathrm{C}_{\text {in }}$ ratio for water transport (Equation 2) [25].

$\frac{C^{l}{ }_{\text {out }}}{C^{l}{ }_{\text {in }}}=\frac{C_{\text {out }}}{C_{\text {in }}} \mathrm{X} \frac{C_{\text {inphenolred }}}{C_{\text {outphenolred }}}$

Where $\mathrm{C}_{\text {inphenolred }}$ and $\mathrm{C}_{\text {outphenolred }}$ represent inlet and outlet phenol red concentrations, respectively.

The plug flow model (Equation 3) was used for calculation of the $P_{\text {eff }}$ values of the drug across the rat ileum [31]. In addition, $\mathrm{k}_{a}$ of the drug (NBV) was calculated using Equation 4 [32]. 
$P_{\text {eff }}(\mathrm{cm} / \mathrm{sec})=\frac{-Q \ln \left(\frac{C^{{ }{ }{ }{ }^{\prime}}}{C{ }{ }_{\text {in }}}\right)}{2 \pi R L}$

$k_{a}(1 / \mathrm{sec})=\frac{\left[1-\left(\frac{C^{l}{ }_{\text {out }}}{C^{l}{ }_{\text {in }}}\right)\right] Q}{\pi r^{2} L}$

Flow rate $(\mathrm{Q}, \mathrm{mL} / \mathrm{sec})$, corrected ratio of outlet to inlet concentration $\left(C^{\prime}{ }_{\text {out }} / C^{\prime}{ }_{\text {in }}\right)$, length of the intestinal segment $(\mathrm{L}, \mathrm{cm})$, and radius of perfused ileal segment $(\mathrm{R}=0.2 \mathrm{~cm})$ were used for calculation of $P_{\text {eff }}$ $[18,22,33,34]$.

The inlet $\left(\mathrm{C}_{\text {in }}\right)$ and outlet $\left(\mathrm{C}_{\text {out }}\right)$ concentrations of phenol red were used to determine net water flux $(\mathrm{NWF})$.

$N W F=\frac{\left(1-\left[\frac{C_{\text {outphenolred }}}{C_{\text {inphenolred }}}\right]\right)}{L} \times Q_{\text {in }}$

A positive NWF value (Eq. 5) indicates secretion of fluid into the segment, while a negative NWF value indicates fluid loss from the mucosal side (lumen)[31].

\subsection{Statistical analysis}

All results were given as mean \pm standard deviation (SD). For multiple comparasion, Kruskal-Wallis test was used. Two tailed non-parametric Mann-Whitney $U$ test was used for two-group comparisons when appropriate, in order to determine differences between the experimental groups. Less than $0.05 p$ value was considered as significant.

Acknowledgements: This study was supported by TUBITAK Project No: 112S292. The authors would like to thank Novartis (Novartis Drug Co., Turkey) for providing metoprolol tartrate. The authors would also like to thank to Ege University, Faculty of Pharmacy, Pharmaceutical Sciences Research Center (FABAL) for high pressure homogenization facilities.

Author contributions: Concept - EHG; Design - EHG, NÜO, MSK.; Supervision - EHG Resource - EHG; Materials NÜO, AY.; Data Collection and/or Processing - EHG, AY, NÜO, MSK.; Analysis and/or Interpretation - AY, NÜO, MSK.; Literature Search - EHG, AY, NÜO Writing - EHG, NÜO, SŞ Critical Reviews - EHG, AY, NÜO, MSK, SŞ.

Conflict of interest statement: The authors declare no conflict of interest.

\section{REFERENCES}

[1] Campbell N, Young ER, Drouin D, Legowski B, Adams MA, Farrell J, Kaczorowski J, Lewanczuk R, Lum-Kwong $\mathrm{MM}$, Tobe S. A framework for discussion on how to improve prevention, management, and control of hypertension in Canada. Can J Cardiol. 2012; 28(3): 262-269.

[2] Ahada A, Aqil M, Kohli K, Sultana Y, Mujeeb M. Enhanced transdermal delivery of an anti-hypertensive agent via nanoethosomes: Statistical optimization, characterization and pharmacokinetic assessment. Int J Pharm. 2013; 443(1-2): 26-38.

[3] Mancia G, Fagard R, Narkiewicz K, Redon J, Zanchetti A, Bohem M, Christiaens T, Cifkova R, De Backer G, Dominiczak A, Galderisi M, Grobbee DE, Jaarsma T, Kirchhof P, Kjeldsen SE, Laurent S, Manolis AJ, Nilsson PM. ESH/ESC guidelines for the management of arterial hypertension: The task force for the management of arterial hypertension of the European Society of Hypertension (ESH) and of the European Society of Cardiology (ESC). J Hypertens. 2013; 31(7): 1281-1357. 
[4] Altoama K, Yassine Mallem M, Thorin C, Betti E, Desfontis J. Effect of nebivolol treatment during pregnancy on the genital circulation, fetal growth and postnatal development in the Wistar rat. Eur J Pharm. 2015; 758: 31-39.

[5] Wang S, Chow M, Zuo Z. An approach for rapid development of nasal delivery of analgesics - Identification of relevant features, in vitro screening and in vivo verification. Int J Pharm. 2011; 420(1): 43-50.

[6] Nigris F, Rienzo M, Schiano C, Fiorito C, Casamassimi A, Napoli C. Prominent cardioprotective effects of third generation beta blocker nebivolol against anthracycline-induced cardiotoxicity using the model of isolated perfused rat heart. Eur J Cancer. 2008; 44(3): 334-340.

[7] Das S, Chaudhary A. Recent advances in lipid nanoparticle formulations with solid matrix for oral drug delivery. AAPS PharmSciTech. 2011; 12(1): 62-76.

[8] Ranpise NS, Korabu SS, Ghodake VN. Second generation lipid nanoparticles (NLC) as an oral drug carrier for delivery of lercanidipine hydrochloride. Colloids Surf B Biointerfaces. 2014; 116: 81-87.

[9] Dery AS, Hamilton LA, Starr JA. Nebivolol for the treatment of heart failure disclosures. Am J Health Syst Pharm. 2011; 68(10): 879-886.

[10] Veverka A, Nuzum DS, Jolly JL. Nebivolol: A thirdgeneration $\beta$-adrenergic blocker. Ann Pharmacother. 2006; 40(78): 1353-1360.

[11] Lennernas H. Animal data: the contributions of the Ussing Chamber and perfusion systems to predicting human oral drug delivery in vivo. Adv Drug Deliv Rev. 2007; 59(11): 1103-1120.

[12] Incecayir T, Tsume Y, Amidon GL. Comparison of the permeability of metoprolol and labetalol in rat, mouse, and Caco-2 cells: use as a reference standard for BCS classification. Mol Pharm. 2013; 10(3): 958-966.

[13] Idkaidek NM, Jilani JA, Mansi IA. Evaluation of hydroxyethyldiclofenac intestinal absorption in rats. Saudi Pharm J. 2005; 13(4): 158-163.

[14] Kheradmandnia S, Vasheghani-Farahani E, Nosrati M, Atyabi F. Preparation and characterization of ketoprofenloaded solid lipid nanoparticles made from beeswax and carnauba wax. Nanomed Nanotechnol Biol Med. 2010; 6(6): 753-759.

[15] Zhang Z, Gao F, Bu H, Xiao J, Li Y. Solid lipid nanoparticles loading candesartan cilexetil enhance oral bioavailability: in vitro characteristics and absorption mechanism in rats. Nanomed Nanotech Biol Med. 2012; 8(5): 740-747.

[16] Li H, Zhao X, Ma Y, Zhai G, Li L, Lou H. Enhancement of gastrointestinal absorption of quercetin by solid lipid nanoparticles. J Control Release. 2009; 133(3): 238-244.

[17] Üstündağ Okur N, Yurdasiper A, Gündoğdu E, Gökçe EH. Modification of solid lipid nanoparticles loaded with nebivolol hydrochloride for improvement of oral bioavailability in treatment of hypertension: Polyethylene glycol versus chitosan oligosaccharide lactate. J Microencaps. 2016; 33(1): 30-42.

[18] Dahan A, Amidon GL. Segmental dependent transport of low permeability compounds along the small intestine due to P-glycoprotein: the role of efflux transport in the oral absorption of BCS class III drugs. Mol Pharm. 2009; 6(1): $19-28$

[19] Heurtault B, Saulnier P, Pech B, Proust JE, Benoit JP. Physico-chemical stability of colloidal lipid particles. Biomaterials 2003; 24(23): 283-300.

[20] Amidon GL, Lennernas H, Shah VP, Crison JR. A theoretical basis for a biopharmaceutic drug classification: the correlation of in vitro drug product dissolution and in vivo bioavailability. Pharm Res. 1995; 12(3): 413-420.

[21] Zakeri-Milani P, Valizadeh H, Azarmi Y, Jalali MB, Tajerzadeh H. Simultaneous determination of metoprolol, propranolol and phenol red in samples from rat in situ intestinal perfusion studies. DARU: J Pharm Sci. 2006; 14(2): 102-108.

[22] Nagare N, Damre A, Singh KS, Mallurwar SR, Iyer S, Naik A, Chintamaneni M. Determination of site of absorption of propranolol in rat gut using in situ single-pass intestinal perfusion. Indian J Pharm Sci. 2010; 72(5): 625-629.

[23] Lennernas H, Abrahamsson B. The use of biopharmaceutic classification of drugs in drug discovery and development: current status and future extension. J Pharm Pharmacol. 2005; 57(3): 273-285.

[24] Kaynak MS, Buyuktuncel E, Caglar H, Sahin S. Determination of regional intestinal permeability of diclofenac and metoprolol using a newly-developed and validated high performance liquid chromatographic method. Trop J Pharm Res. 2015; 14(1): 163-170. 
[25] Kim JS, Mitchell S, Kijek P, Tsume Y, Hilfinger J, Amidon GL. The suitability of an in situ perfusion model for permeability determinations: utility for BCS class I biowaiver requests. Mol Pharm. 2006; 3(6): 686-694.

[26] Anton N, Benoit JP, Saulnier P. Design and production of nanoparticles formulated from nano-emulsion templates-a review. J Control Release. 2008; 128(3): 185-199.

[27] Yang Z, Gan G, Sawchuk RJ. Correlation between net water flux and absorptive clearance determined from in situ intestinal perfusion studies does not necessarily indicate a solvent drag effect. J Pharm Sci. 2007; 96(3): 517-521.

[28] Yang T, Sheng HH, Fen NP, Wei H, Wang Z, Wang C. Preparation of andrographolide-loaded solid lipid nanoparticles and their in vitro and in vivo evaluations: Characteristics, release, absorption, transports, pharmacokinetics, and antihyperlipidemic activity. J Pharm Sci. 2013; 102(12): 4414-4425.

[29] Sangsena Y, Wiwattanawongs K, Likhitwitayawuid K, Sritularak B, Wiwattanapatape R. Modification of oral absorption of oxyresveratrol using lipid based nanoparticles. Coll Surf B. 2015; 131: 182-190.

[30] Gonçalves LMD, Maestrelli F, Manelli L, Ghelardini C, Almeida AJ, Mura P. Development of solid lipid nanoparticles as carriers for improving ora bioavailability of glibenclamide. Eur J Pharm Biopharm. 2016; 102: 4150.

[31] Fagerholm U, Johansson M, Lennernas H. Comparison between permeability coefficients in rat and human jejunum. Pharm Res. 1996; 13(9): 1336-1342.

[32] Gao H, Wang M, Sun D, Sun S, Sun C, Liu J, Guan Q. Evaluation of the cytotoxicity and intestinal absorption of a self-emulsifying drug delivery system containing sodium taurocholate. Eur J Pharm Sci. 2017; 106: 212-219.

[33] Komiya I, Park JY, Kamani A, Ho NFH, Higuchi WI. Quantitative mechanistic studies in simultaneous fluid-flow and intestinal-absorption using steroids as model solutes. Int J Pharm. 1980; 4(3): 249-262.

[34] Patel JR, Barve KH. Intestinal permeability of lamivudine using single pass intestinal perfusion. Indian J Pharm Sci. 2012; 74(5): 478-433.

This is an open access article which is publicly available on our journal's website under Institutional Repository at http://dspace.marmara.edu.tr. 